Document downloaded from:

http://hdl.handle.net/10251/90967

This paper must be cited as:

Hueso González, J.; Raboso García-Baquero, D.; Ernst, C.; Schmitt, D.; Boria Esbert, VE.; Gimeno Martinez, B.; Taroncher Calduch, M.... (2013). Optimized Multipactor-Resistant Wedge-Shaped Waveguide Bandpass Filters. IEEE TRANSACTIONS ON PLASMA SCIENCE. 41(8):2135-2144. doi:10.1109/TPS.2013.2253134

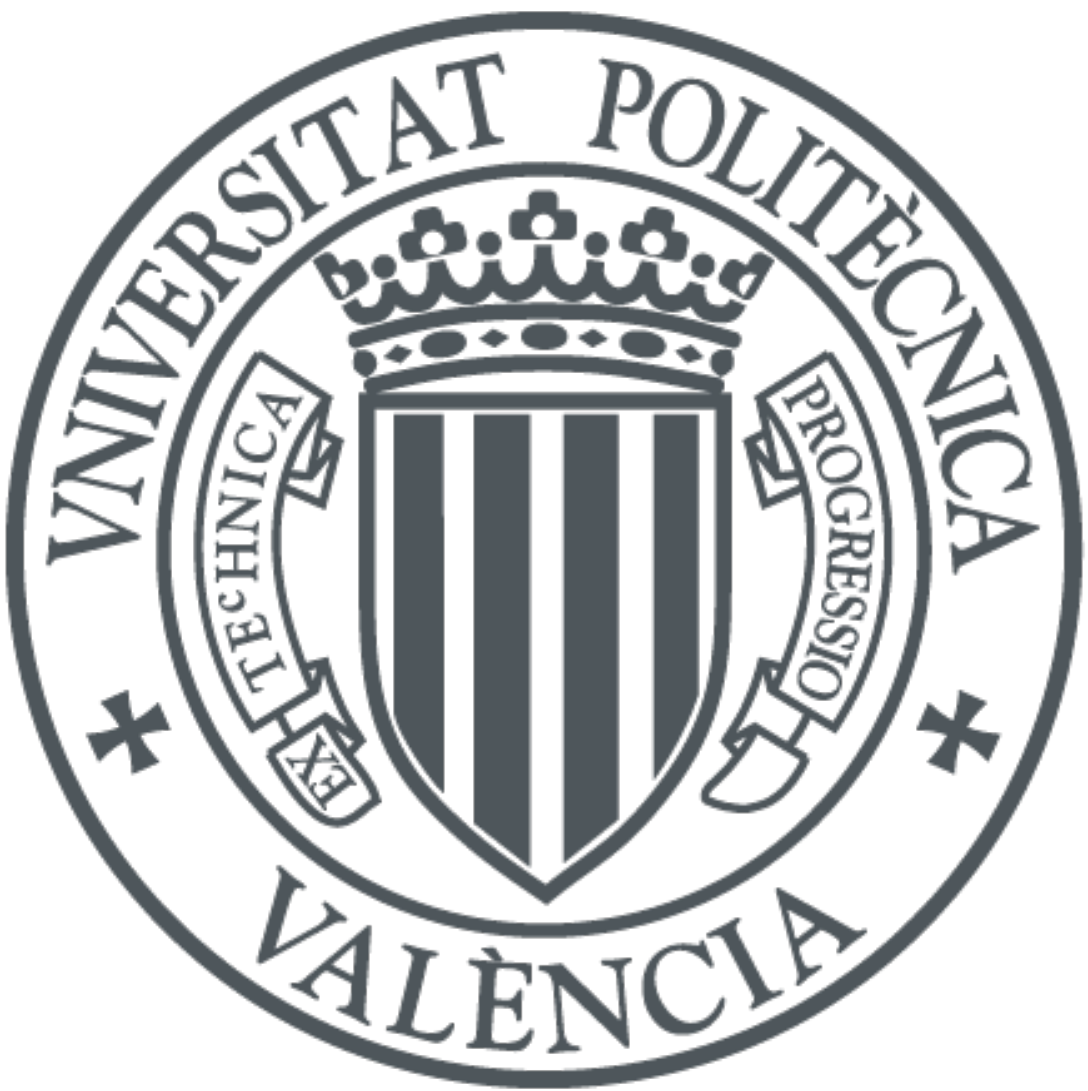

The final publication is available at

http://doi.org/10.1109/TPS.2013.2253134

Copyright

Additional Information 


\title{
Optimized Multipactor-Resistant Wedge-Shaped Waveguide Bandpass Filters
}

\author{
Jaime Hueso, David Raboso, Christoph Ernst, Member, IEEE, Dietmar Schmitt, \\ Vicente E. Boria, Senior Member, IEEE, Benito Gimeno, Member, IEEE, \\ Mariam Taroncher, Student Member, IEEE, and Carlos Vicente, Member, IEEE
}

\begin{abstract}
Wedge-shaped waveguides present a certain advantage with respect to rectangular waveguides regarding their resistance to multipactor discharges. In this paper, the optimal configuration for the wedge geometry is investigated based on theoretical results, a precise multipactor prediction tool and previous experience. In addition, design rules are presented, which allow to achieve comparable electrical performances of wedgeshaped filters to rectangular waveguide filters, whilst at the same time improving the multipactor-free power range. As a proof of concept, two three-pole bandpass filters with equivalent electrical characteristic of $150 \mathrm{MHz}$ bandwidth, centered at $12 \mathbf{~ G H z}(\mathbf{K u}$ band), and the same $Q$ factor have been designed, manufactured and tested. The first design is based on conventional rectangular waveguide technology, while the second one has non-parallel broadside walls (wedge-shaped cross section). The multipactor power threshold and RF performance of the filters have been measured in order to validate the improvements achievable employing wedge-shaped resonators.
\end{abstract}

Index Terms-Bandpass filters, circuit synthesis, microwave filters, multipactor, space technology, vacuum breakdown.

\section{INTRODUCTION}

$\mathbf{R}$ ECTANGULAR waveguide filters widely used in spaceborne hardware are prone to suffer multipactor (MP) discharges [1], [2] in high power applications. As a consequence, the electrical signal transmission may be compromised and even physical damage may occur to the hardware. Practical examples can be observed at multiplexer ports, antenna feeds and amplifier outputs on-board communication satellites. Thus, costly MP testing is often needed for certifying the flight qualification of all power-critical space hardware, and therefore power limitations have to be set to avoid risks. As an alternative, "wedge-shaped" waveguide cross sections as the one in Fig. 1 have a higher resistance to MP RF breakdown [3]-[6]. The wedge-shaped topology is composed of symmetrically inclined top and bottom walls and straight vertical side-walls (see Fig. 1). Hence, the height of the waveguide changes linearly from the narrow side-wall to the broad one [7]. For inclination angle $(\alpha)$-values between $0^{\circ}$ to $60^{\circ}$, the electrical properties of the fundamental mode of the

J. Hueso is with the European Patent Office (EPO), The Netherlands. Email: jhuesogonzalez@epo.org. D. Raboso, C. Ernst and D. Schmitt are with the European Space Agency, Noordwijk, The Netherlands. V. E. Boria and M. Taroncher are with the Departamento de Comunicaciones - iTEAM, Universidad Politécnica de Valencia, Spain. B. Gimeno is with the Departamento de Física Aplicada - ICMUV, Universidad de Valencia, Spain. C. Vicente is with the Aurora Software and Testing S.L., Universidad Politécnica de Valencia, 46022 Valencia, Spain.

Manuscript received March 03, 2013

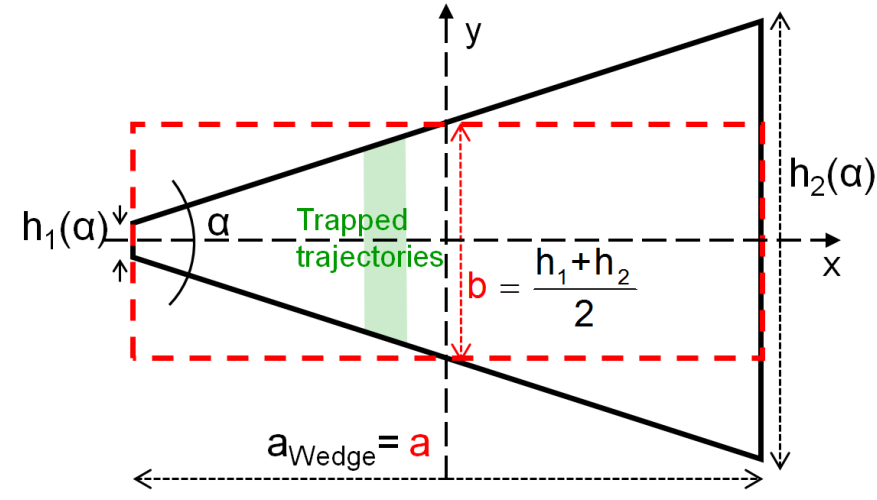

Fig. 1. Cross-section view of a wedge-shaped (black-solid line) and a rectangular waveguide (red-dashed line) with the same cross-section area. Shaded in green, region within the wedge-shaped geometry with trapped electron trajectories.

structures are similar to the rectangular waveguide ones, which poses the question whether they might be interchangeable in certain applications. However, the inclination between the top and bottom walls can perturb the MP resonance behavior [5], [6], which is the main advantage sought with respect to a rectangular geometry. We define a wedge-shaped waveguide as a microwave transmission line with such a constant cross section in the propagation direction. At $60^{\circ}$, the cross section is similar to an equilateral triangle, with a considerably different fundamental mode [8]. Hence, inclinations of $60^{\circ}$ and above are less interesting for this study.

In [6], software tools that allow us to perform the RF design and analysis but also to perform a MP analysis of wedgeshaped waveguide were presented, which have been integrated into FEST $^{3 \mathrm{D}}$ (Full-Wave Electromagnetic Simulation Tool) [9]. An electromagnetic solver that implements the Boundary Integral - Resonant Mode Expansion (BI-RME) method [10], [11] allows to calculate the electromagnetic fields in any wedge-shaped geometry. A design synthesis method [12], [13] was adapted to allow deriving dimensions of bandpass filters with arbitrary wedge-shaped geometries. Additionally, a MP prediction tool was implemented based on the computation of the effective electron trajectories. This allows to analyze the secondary electron growth in the device, and to provide a prediction of the MP discharge risk [5]. A preliminary attempt to use these techniques for designing a hardware filter prototype was reported in [6], and an enhancement of $1 \mathrm{~dB}$ in the MP RF threshold value was determined by test. However, 
neither rigorous design guidelines were available, nor was it known how to achieve optimal power handling capabilities.

In this paper, new concepts are applied to the design procedure in order to reach a substantial enhancement of the MP behavior of wedge-shaped filters. First, a parametric analysis is performed in order to derive the optimal inclination angle for the wedge-shaped topology. Moreover, the width of the inner stages of the filter is increased so that the out-of-band response of the filter becomes comparable to the rectangular one. Refinements of the manufacturing process are proposed in order to cope with the additional complexity of the wedgeshaped topology. Finally, a three-pole bandpass filter centered at $12 \mathrm{GHz}$ (Ku band) with $150 \mathrm{MHz}$ bandwidth and a wedgeshaped cross-section geometry has been designed and two copies of it (one with gold plating, the other in bare aluminum) manufactured in ESA-ESTEC, The Netherlands. As a reference for performing a comparative study, an equivalent rectangular waveguide quasi-inductive filter with the same electrical behavior and Q factor has been designed as well (a gold-plated and an aluminum version were manufactured). The four devices have been tested in the Payload Systems Laboratory at ESA-ESTEC, thus verifying the predicted improvements in terms of MP free range. Such verification is useful for increasing the maturity of this technology for a potential application in space.

The paper is organized as follows: Section II presents the steps leading to the optimized design of wedge-shaped filters, namely the choice of the inclination angle in Section II-A, the improvement of the out-of-band response in Section II-B, and the solution of some mechanical manufacturing issues in Section II-C. The manufactured bandpass filters, which take into account all these issues, are presented in Section III. Section III-A includes the specifications and the physical description of the filters, and the measurements of the frequency responses are shown in Section III-B. In Section III-C, the focus is set on the MP prediction techniques applied to these filters, and they are compared with the MP threshold measurements obtained in the laboratory. Section IV summarizes all design rules derived and applied to the hardware presented in this paper, and discusses the potentials of this new waveguide geometry.

\section{WedGe-Shaped GeOMetry Studies}

\section{A. Inclination Angle}

The selection of the most suitable $\alpha$-values between the top and the bottom plates (see Fig. 1) is an essential design criterion for the wedge-shaped filter. Considering an operating frequency of $12 \mathrm{GHz}$, a reference rectangular waveguide with width $a=19.05 \mathrm{~mm}$ (WR75) and height $b=6.26 \mathrm{~mm}$ has been chosen. This particular height value is equal to the average height of the wedge-shaped waveguides applied in the filter design (described in Section III-A), hence it appeared to be a better choice for comparison purposes than the standard WR75 height. Keeping the width constant and an equal crosssection area (i.e. the average height of each wedge-shaped waveguide is $b=6.26 \mathrm{~mm}$ ), as in Fig. 1, several waveguide cross sections with a sweep of inclination angles between $0^{\circ}$ and $60^{\circ}$ can be obtained by selecting the appropriate values of $h_{1}(\alpha)$ and $h_{2}(\alpha)$. Note that this is only possible up to $\alpha \approx 35^{\circ}$ for the given reference waveguide dimensions. A lower boundary $h_{1, \min } \geq 0.2 \mathrm{~mm}$ has been set due to manufacturing constraints. Once it is reached, higher $\alpha$-values result in average heights larger than $b$ and consequently larger cross-section areas. Hence, the inclinations over $35^{\circ}$ are not exactly comparable with the rectangular case, but they are kept in the analysis for completeness.

In a first step, for each wedge-shaped cross section, a region is identified within where free electrons could follow a resonant path between the top and bottom inclined walls. The necessary condition to enable such a resonant path is that the excited electromagnetic fields generate a Miller force that exactly compensates the centrifugal effect experienced by the accelerated free electrons [4]. More precisely, [4] proposes an equation to determine such a region in annular-section waveguides. Annular-section waveguides have a cross section formed by a circular sector delimited by an inner and an outer arc with radii $R_{1}$ and $R_{2}$. The inner and outer radii would correspond to the side-walls of the analogous wedgeshaped waveguide. Following this approach, a disk of potential critical radii, with boundaries $R_{\text {crit,min }}$ and $R_{\text {crit,max }}\left(R_{1}<\right.$ $R_{\text {crit,min }}<R_{\text {crit,max }}<R_{2}$ ), can be identified. Assuming the validity of the analogy between the annular-section and wedgeshaped waveguides [5], we identify a disk area delimited by the critical radii close to the center of the wedge-shaped cross section (see green highlighted region in Fig. 1). Electrons launched from the top or the bottom walls of this area are likely to get trapped in the mentioned resonant trajectories, thus enabling the possibility of a sustained electron growth. A voltage distribution can then be calculated for the different radii of the critical region [5], [6] by means of a line integral of the electric field following an arc of circumference delimited by the wedge top and bottom walls. An arbitrary input power can be selected for calculating the voltage, which in this case is equal to $1 \mathrm{~W}$. MP simulation results presented in [5] show that the most sensitive radius to MP discharge effects is located close to the position of the calculated region with a higher radius. Following this procedure, the voltage in the estimated critical radius has been plotted (dark-blue-solid curve) for all wedge-shaped waveguides considered in this study, as it can be seen in Fig. 2.

The boundaries of the trapped trajectory region (as indicated by the green rectangle in Fig. 1) are depicted in Fig. 2 as greendashed curves, assigned to the right axis. The limits are always on the negative side of the $x$-axis, i.e. closer to the narrow sidewall [5]. The green-dotted line in Fig. 2 presents the position chosen for the calculation of the voltage where the maximum MP discharge is expected. The grey-shaded region shows the $\alpha$-values for which the average height exceeds the value of $b$. The voltage curve shows a minimum value for $\alpha \approx 30^{\circ}$, which means that the trapped electrons of this wedge-shaped waveguide will experience the smallest voltage values of all simulated cases. Hence, it is expected that the wedge-shaped waveguide with this inclination angle is the most resistant to MP discharges. Consequently, an inclination of $\alpha=30^{\circ}$ has been chosen for the optimal design of a MP resistant 


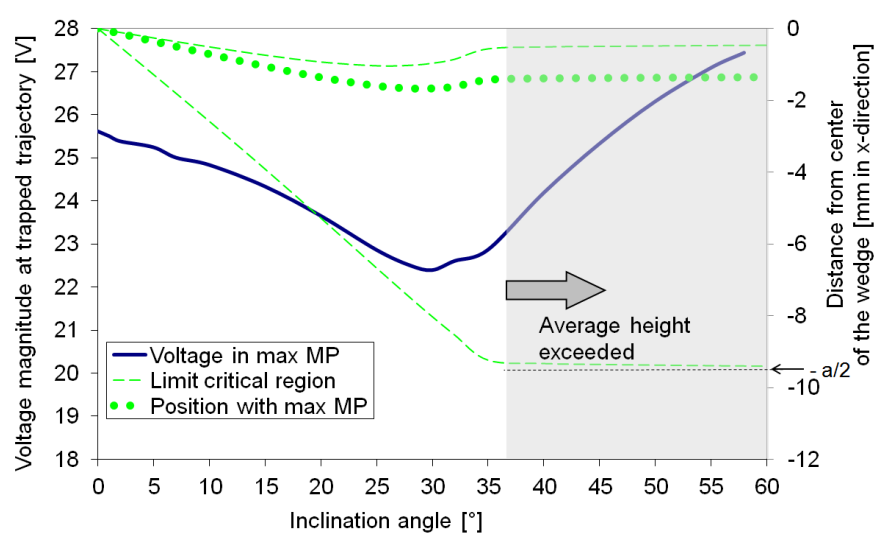

Fig. 2. Voltage (for $1 \mathrm{~W}$ RF input power) in the critical MP radius for all the wedge-shaped waveguides considered in the inclination angle sweep: constant width $a_{\text {Wedge }}=a$ and, whenever possible, constant cross-section area. A reference rectangular waveguide with dimensions $a=19.05 \mathrm{~mm}$ and $b=6.26 \mathrm{~mm}$ has been selected. Operating frequency $12 \mathrm{GHz}$. Green curves are assigned to the right axis and represent the location of the trapped trajectories region.

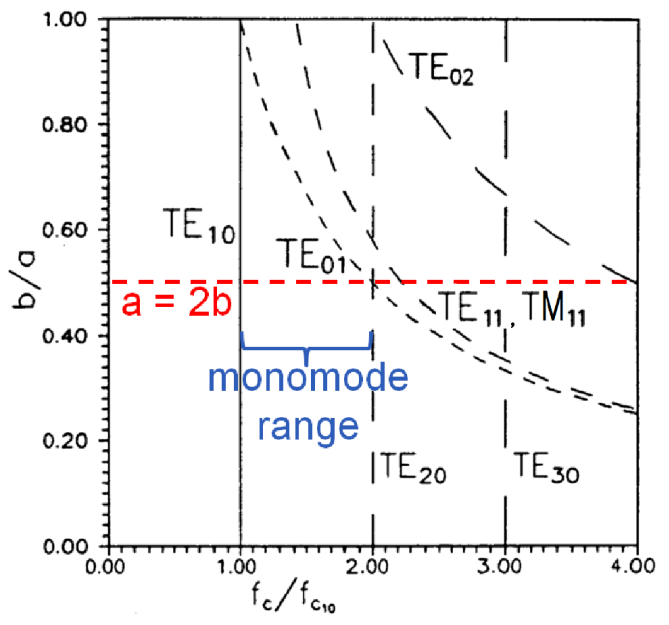

Fig. 3. Mode chart of a rectangular waveguide. Cutoff frequencies of the different modes in terms of the ratio between the height $b$ and the width $a$.

wedge-shaped waveguide bandpass filter. This is different to [6], where the angle was $19^{\circ}$. Experimental MP measurements verify the expected performance improvement, as it will be shown later in the paper.

\section{B. Out-of-band Frequency Response}

In rectangular waveguides, a typical design criterion aims for minimal losses for a given operating frequency. This is the case when the rectangular cross section tends to be square, i.e. $b / a \rightarrow 1$. At the same time, it is wished to have a broad monomode frequency range. The operating frequency is usually chosen slightly above the cutoff frequency of the fundamental mode. The difference between the operating frequency and the one of the second order mode determines the monomode bandwidth. As it can be seen in Fig. 3, for values of $b / a \leq 0.5$, the cutoff frequency of the second order mode $\mathrm{TE}_{20}$ keeps a constant value. If $b / a>0.5$, the $\mathrm{TE}_{01}$ mode becomes the second order mode, and the

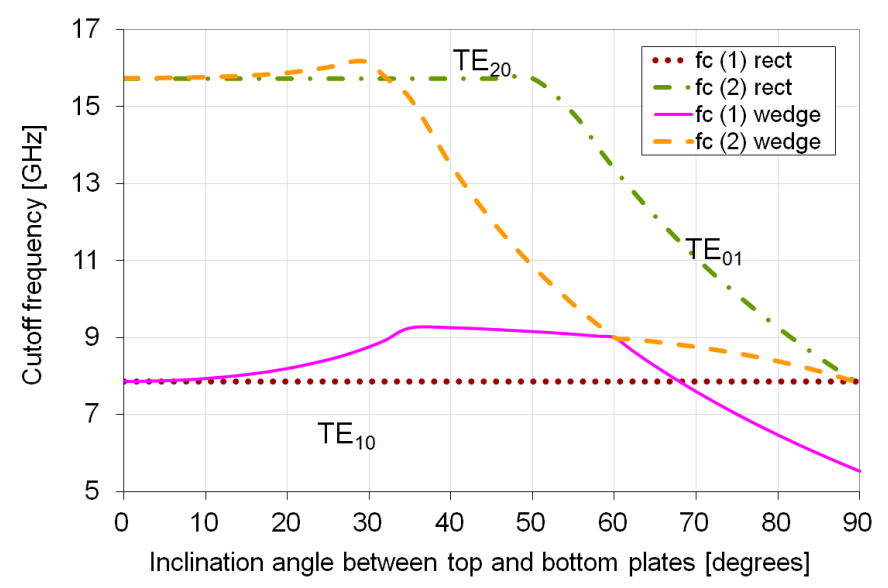

Fig. 4. Cutoff frequencies of the first two propagating modes of a wedgeshaped waveguide inclination angle sweep: constant width $a_{\text {Wedge }}=a$ and, whenever possible, constant cross-section area. Values are compared to the ones of the reference rectangular waveguide with dimensions $a=19.05 \mathrm{~mm}$ and $b=6.26 \mathrm{~mm}$.

monomode bandwidth systematically decreases with the $b / a$ ratio. Therefore, the optimal size ratio is 0.5 , since it offers the less possible losses without sacrificing monomode bandwidth.

A similar cutoff frequency analysis can be done for wedgeshaped waveguides. In order to allow a direct comparison with the reference rectangular waveguide, the same approach followed in Section II-A (but considering wedge-shaped waveguides with inclination angles up to $\alpha=90^{\circ}$ ) has been reused here. The shape of the electromagnetic fields of the first two propagating modes and their cutoff frequencies $\left(f_{\mathrm{C}}(1)\right.$ and $f_{\mathrm{C}}(2)$ ) were analyzed, and the results shown in Fig. 4 (magenta solid and orange dashed curves) over the inclination angle. As a reference, the $f_{\mathrm{C}}(1)_{\text {rect }}$ and $f_{\mathrm{C}}(2)_{\text {rect }}$ of the corresponding rectangular waveguide were also plotted (brown dotted and green dash-dotted curves). The indices 1 and 2 are dynamically assigned to the first two propagating modes, in terms of the ascending cutoff frequency value order. We can observe that there are relatively sudden changes in the slopes of the curves: at $\alpha \approx 30^{\circ}$ for $f_{\mathrm{C}}(2)_{\text {wedge }}, \alpha \approx 50^{\circ}$ for $f_{\mathrm{C}}(2)_{\text {rect }}$ and $\alpha \approx 60^{\circ}$ for $f_{\mathrm{C}}(1)_{\text {wedge }}$, caused by changes in the mode order. For small inclination angles $\left(\alpha<35^{\circ}\right)$, the wedge-shaped waveguide fundamental mode has a $\mathrm{TE}_{10}$-like electric field pattern, and also similar $f_{\mathrm{C}}$ values to those of the rectangular one. For $35^{\circ}<\alpha<60^{\circ}$, the field patterns have a stronger distortion with respect to the $\mathrm{TE}_{10}$, and the differences between $f_{\mathrm{C}}(1)_{\text {wedge }}$ and $f_{\mathrm{C}}(2)_{\text {wedge }}$ are gradually reduced to zero. This point is reached at $\alpha \approx 60^{\circ}$, which corresponds to a cross section with equilateral triangular shape [8]. Within the range of $\alpha$-values of Fig. 4, and especially for $\alpha<30^{\circ}$, it can be stated that the first two propagating modes of the wedge-shaped waveguide have a similar behavior to the rectangular waveguide ones. However, the wedgeshaped cutoff frequency $f_{\mathrm{C}}(1)$ is consistently higher than the rectangular one for the range of interest. This would have a negative effect on the design of the filter resonators, since it shifts the filter repetition band closer to the pass-band [6].

A solution to avoid this problem is to gradually adapt the wedge-waveguide width $a_{\text {Wedge }} \neq a$ in order to obtain a $f_{\mathrm{C}}(1)$ 


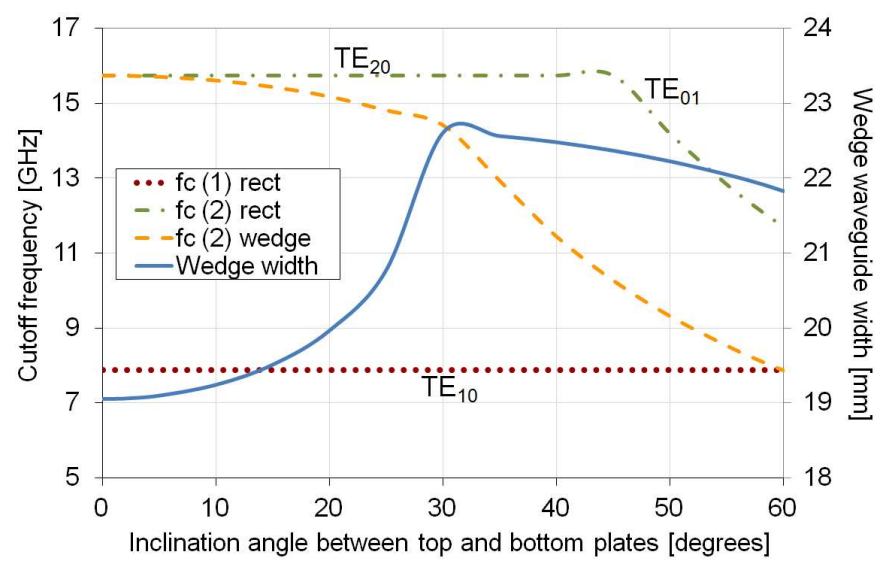

Fig. 5. Cutoff frequencies of the first two propagating modes of a wedge-shaped waveguide inclination angle sweep: constant cutoff frequency $f_{\mathrm{C}}=7.869 \mathrm{GHz}$ and, whenever possible, constant average height. Values are compared to the ones of the reference rectangular waveguide with dimensions $a=19.05 \mathrm{~mm}$ and $b=6.26 \mathrm{~mm}$. Solid blue curve represents the values of $a_{\text {Wedge }}$.

equal to the one of the reference rectangular waveguide for each given $\alpha$-case. The average height of the waveguide is still kept equal to the rectangular $b$. The result of this exercise is shown in Fig. 5. The solid blue line represents the required variation of the width with the inclination angle (assigned to the right-hand side axis of ordinates). Its value is always higher than in the rectangular waveguide case $\left(\alpha=0^{\circ}\right)$, reaching a maximum value between $30^{\circ}$ and $40^{\circ}$. The criterion of equal cross-section areas cannot be kept here anymore, but the average gap has been chosen as the preferred criterion for the comparison. The differences in the cross-section values keep in any case small, since the variation in the $a_{\text {Wedge }}$-values is less than $15 \%$. For the particular analysis of Fig. 5, it must be noted that the maximum applicable angle is around $37^{\circ}$, since otherwise more than one mode would be able to propagate at the working frequency of $12 \mathrm{GHz}$ for the chosen waveguide. The reduction in the monomode bandwidth can be however acceptable for $\alpha<30^{\circ}$.

The analysis of the voltages in the electron trapped trajectories of Section II-A can be then repeated for the new approach of keeping a constant $f_{\mathrm{C}}$, since it seems to be more convenient for wedge-shaped filters (see Fig. 6). Taking into account that both plots (Fig. 2 and Fig. 6) are represented with the same axes, the main difference is that the voltage curve experiments a deeper minimum here. This is due to the fact that $a_{\text {Wedge }}$ is increased gradually with $\alpha$, which increases the cross-section area and reduces the voltage for the same normalized input power. The most important conclusion is that the minimum still lies at $30^{\circ}$.

Combining the results from this and the previous section, it can be concluded that $\alpha=30^{\circ}$ is the most convenient angle for designing a bandpass filter with a wedge-shaped topology that has an optimal MP behavior, while keeping the electrical performance of the rectangular reference case.

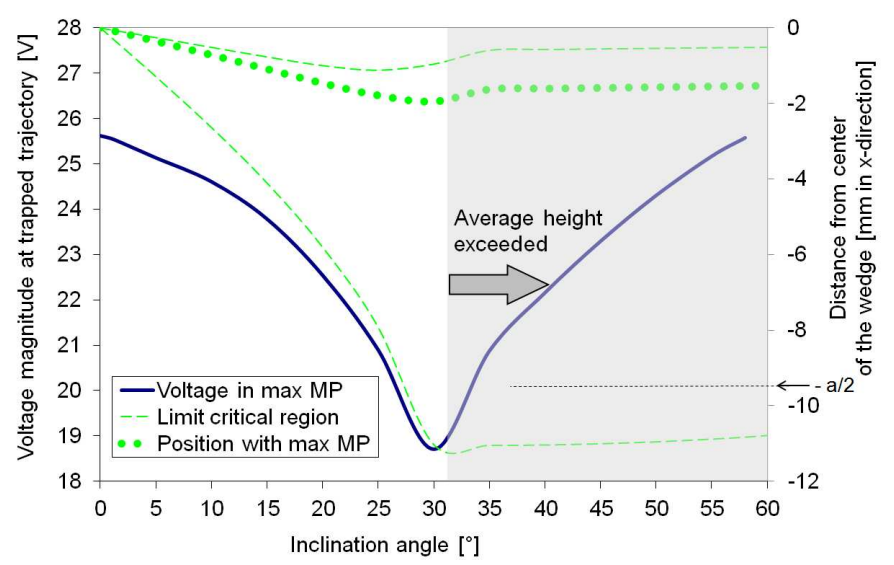

Fig. 6. Voltage (for $1 \mathrm{~W}$ RF input power) in the critical MP radius for all the wedge-shaped waveguides considered in the inclination angle sweep: constant $f_{\mathrm{C}}$ and, whenever possible, constant average height. A reference rectangular waveguide with dimensions $a=19.05 \mathrm{~mm}$ and $b=6.26 \mathrm{~mm}$ has been selected. Operating frequency $12 \mathrm{GHz}$. Green curves are assigned to the right axis and represent the location of the trapped trajectories region.

\section{Manufacturing Issues}

Waveguide filters are usually manufactured in two halves, which are typically joined together with screws. The junction plane is the cut where the internal cavities of the filter make contact, which is essential for the correct electrical behavior of the device. The pressure plane is the cut where the screws that mechanically hold both halves together make contact. In rectangular waveguide filters, it is common that both junction and pressure plane are the same one, so that increasing the pressure on the screws will directly translate in junction plane pressure, avoiding potential electrical contact problems. However, when manufacturing a wedge-shaped filter, an important aspect is the additional complexity of having non-parallel top and bottom walls, which are also not perpendicular to the vertical side walls. One possible solution for the wedgeshaped case is to drill the wedged cavity shapes in one single metallic block, and use a simple flat metallic plate for closing the structure. This solution almost divides by two the manufacturing complexity, since only one of the halves has to be machined with high accuracy. However, this manufacturing process caused problems in our previous designs, since the junction plane was not the same as the pressure plane of the screws, resulting in a bad contact that disturbed the filter frequency response [6]. Therefore, we adopted here an approach based on two completely symmetrical halves, where both the junction and the screw pressure plane were horizontal. This plane is represented by the $x$-axis in Fig. 1 and is also shown in Fig. 7. Although the machining process has to be performed twice, there is no substantial increase in complexity, since both halves are completely identical.

Another improvement with respect to the approach followed in [6] is that the filters, two rectangular and two wedgeshaped ones, have been manufactured in-house (ESA-ESTEC) in a common milling machining process. Hence, the same materials have been used, which ensures a fair comparison procedure. For the wedge-shaped filters, a relatively coarse mill with $1.5 \mathrm{~mm}$ curvature radius had to be used (instead of a 


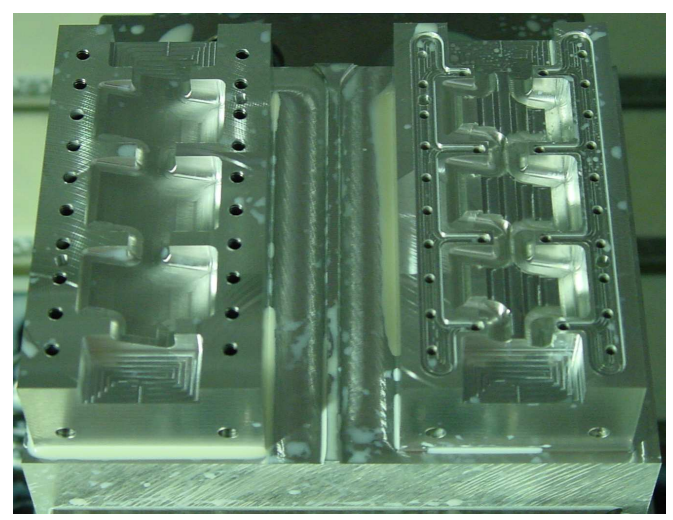

Fig. 7. Wedge-shaped filter halves during the manufacturing process.

TABLE I

REQUIREMENTS OF THE BANDPASS FILTERS

\begin{tabular}{|c|c|}
\hline Parameter & Value \\
\hline Center frequency [GHz] & 12 \\
\hline Bandwidth [MHz] & 150 \\
\hline Order of the filter & 3 \\
\hline Return loss & 25 \\
\hline
\end{tabular}

sharp-end one) due to the non-parallel plates, which generates some rounded corners in said filters (cf. Fig. 7). Since the drill size was known, this was considered in the fine-tuning procedure of the dimensions before starting the manufacturing process.

From a MP test point of view, it has to be ensured that there is a sufficient electron seeding in the inner part of the filter. A ${ }^{90} \mathrm{Sr} \beta$-emitting radioactive source has been used for this purpose, which produces two different emitters: ${ }^{90} \mathrm{Sr}$ (half-life of 28 years) and ${ }^{90} \mathrm{Y}$ (half-life of just 2 days), with a maximum kinetic energy per electron of $0.546 \mathrm{MeV}$ and $2.28 \mathrm{MeV}$, respectively. The maximum penetration depth of the ${ }^{90} \mathrm{Y}$ electrons in an aluminum wall is around $5.25 \mathrm{~mm}$. Thus, the external metallic walls of both filter halves have to be considerably thinner than this constraint (in this case a thickness value of $1.5 \mathrm{~mm}$ was chosen).

\section{IMPLEMENTED DESIGNS}

\section{A. Designed Filters}

Both filter designs have to meet the same electrical specifications of Table I, which are typical for space applications: $12 \mathrm{GHz}$ center frequency and $150 \mathrm{MHz}$ bandwidth. The input and outputs are WR75 waveguides and the order of the filters has been fixed to three, which corresponds to the number of resonators.

They have been designed with the help of $\mathrm{FEST}^{3 \mathrm{D}}$, which implements an automatic rectangular-filter dimensionalsynthesis procedure described in [12] and [13]. The design of the wedge-shaped filter is slightly more complex, since additional wedge-shape discontinuity transfer-function computations are required. The wedge-shaped filter was however designed first, with an inclination angle of $30^{\circ}$, as established in Sections II-A and II-B. This inclination is kept constant along all the intermediate stages of the filter, excepting the

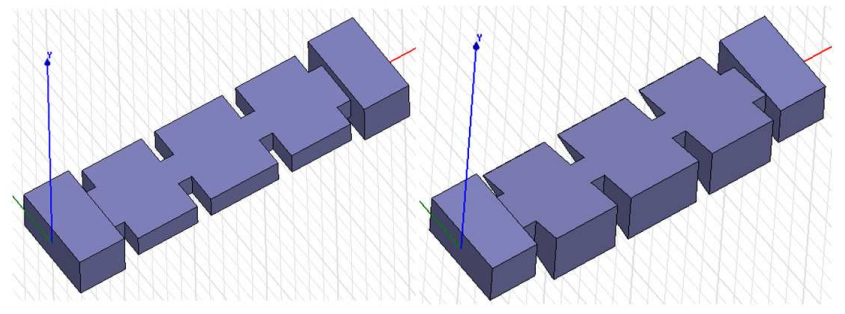

Fig. 8. 3D views of the quasi-inductive rectangular (left) and the wedgeshaped (right) filters.

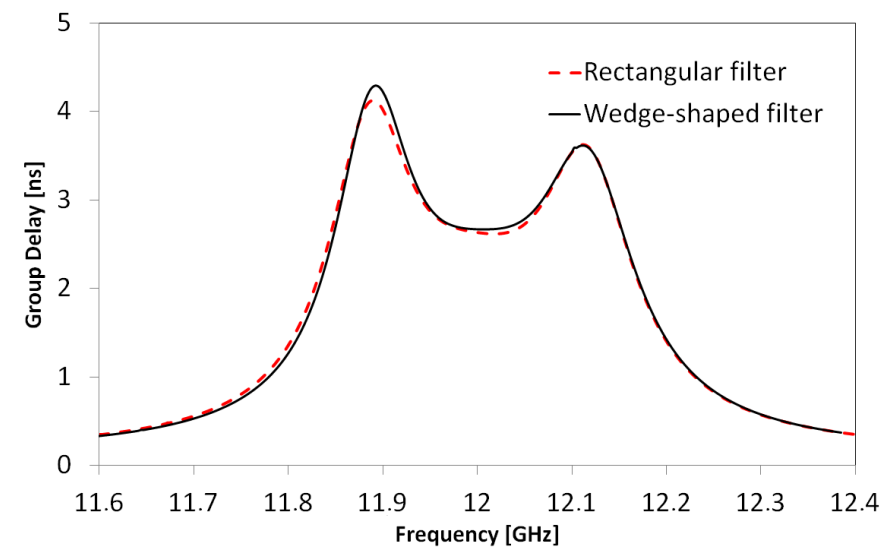

Fig. 9. Comparison of the group delays of both structures. Simulation with FEST $^{3 \mathrm{D}}$.

input and output waveguides (right-hand side of Fig. 8). The minimum gap has been fixed to the manufacturing limit $h_{1, \text { min }}=0.2 \mathrm{~mm}$. Larger gaps would result in a RF input power threshold for MP discharges exceeding the measuring range of the available equipment. The resulting maximum height is $h_{2, \max }=12.323 \mathrm{~mm}$. The width has been iteratively calculated to have the same $f_{\mathrm{C}}$ value as the WR75 fundamental mode.

The rectangular waveguide filter is a symmetric structure, originally (as designed with $\mathrm{FEST}^{3 \mathrm{D}}$ ) purely inductive: $\mathrm{H}$ plane and constant height. However, the height of the intermediate stages of the filter has to be decreased (left-hand side of Fig. 8) to perfectly match the $\mathrm{Q}$ factor of the wedge-shaped filter (whose simulated value is 5440) and to achieve comparable frequency responses. The reduced height value obtained for this quasi-inductive filter is $b_{\text {rect }}=5.05 \mathrm{~mm}$. A comparison of their simulated group delay can be seen in Fig. 9. The $Q$ factor is directly linked to the losses in the whole structure, so both filters have as well the same insertion losses due to the finite conductivity of the materials at $12 \mathrm{GHz}(0.18 \mathrm{~dB}$ for silver with a conductivity of $6.1 e 7 \mathrm{~S} / \mathrm{m}$ ). Please note that, due to the Q-factor optimization, the reduced height of the rectangular filter is slightly smaller than the average height of the wedgeshaped filter $\left(b_{\text {rect }}=5.05 \mathrm{~mm}<\frac{h_{2, \max }+h_{1, \min }}{2}=6.26 \mathrm{~mm}\right)$. This ensures a fairer comparison between the rectangular and wedge-shaped filters than in previous works [6].

A comparison of the cross sections of both filters is depicted in Fig. 10, and all dimensions are listed in Tables II and III. Both filters have horizontal symmetry, and are also symmetric 


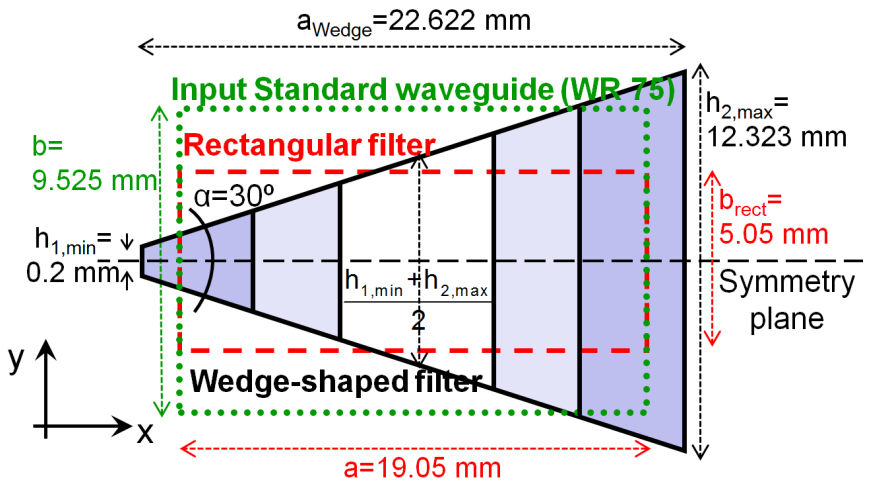

Fig. 10. Comparative detail of the cross sections of the two considered filters. The mean wedge height is slightly different from the rectangular filter height $b_{\text {rect }}$ in order to match the Q factors.

TABLE II

DIMENSIONS OF THE RECTANGULAR WAVEGUIDE BANDPASS FILTER

\begin{tabular}{|c|c|c|c|}
\hline WG & $\mathrm{a}[\mathrm{mm}]$ & $\mathrm{b}[\mathrm{mm}]$ & $1[\mathrm{~mm}]$ \\
\hline Input & 19.05 & 9.525 & 10 \\
\hline Iris 1 & 9.744 & 5.05 & 3 \\
\hline Cavity 1 & 19.05 & 5.05 & 13.275 \\
\hline Iris 2 & 6.151 & 5.05 & 3 \\
\hline Cavity 2 & 19.05 & 5.05 & 15.062 \\
\hline
\end{tabular}

with respect to a perpendicular plane to the propagation energy direction placed in the center of each of both filters. Furthermore, the rectangular waveguide filter has also vertical symmetry (see Fig. 10).

Additionally, and with the help of $\mathrm{FEST}^{3 \mathrm{D}}$ software tool, a tolerance analysis was performed before proceeding with the filters manufacturing process. For a three-pole bandpass filter with the required characteristics of this design, a value of $8.7 \mu \mathrm{m}$ comes out as the dimension tolerance requested to guarantee around $25 \mathrm{~dB}$ of return loss, while $12.325 \mu \mathrm{m}$ is required to achieve $20 \mathrm{~dB}$ return loss. Then, a tolerance value of $10 \mu \mathrm{m}$ was fixed for the manufacturing procedures. In the first pair of filters, the pieces are manufactured in aluminum plus a gold-plating layer of around $10 \mu \mathrm{m}$ thickness, in order to ensure an almost lossless behavior of the structure. In the second pair, just bare aluminum was used, which also acts as the plating.

\section{B. S-Parameters Measurements}

The manufactured filters (see gold-plated pair in Fig. 11) were measured in the ESA-ESTEC Payload Systems laboratory. A comparison between the measured and the simulated

TABLE III

DIMENSIONS OF THE WEDGE-SHAPED WAVEGUIDE BANDPASS FILTER

\begin{tabular}{|c|c|c|c|c|}
\hline WG & $\mathrm{a}[\mathrm{mm}]$ & \multicolumn{2}{|c|}{$\mathrm{b}[\mathrm{mm}]$} & $1[\mathrm{~mm}]$ \\
\hline Input & 19.05 & \multicolumn{2}{|c|}{9.525} & 10 \\
\hline & & $h_{2}$ & $h_{1}$ & \\
\hline Iris 1 & 10.279 & 9.016 & 3.507 & 3 \\
\hline Cavity 1 & 22.622 & 12.323 & 0.2 & 13.99 \\
\hline Iris 2 & 6.766 & 8.074 & 4.449 & 3 \\
\hline Cavity 2 & 22.622 & 12.323 & 0.2 & 15.515 \\
\hline
\end{tabular}

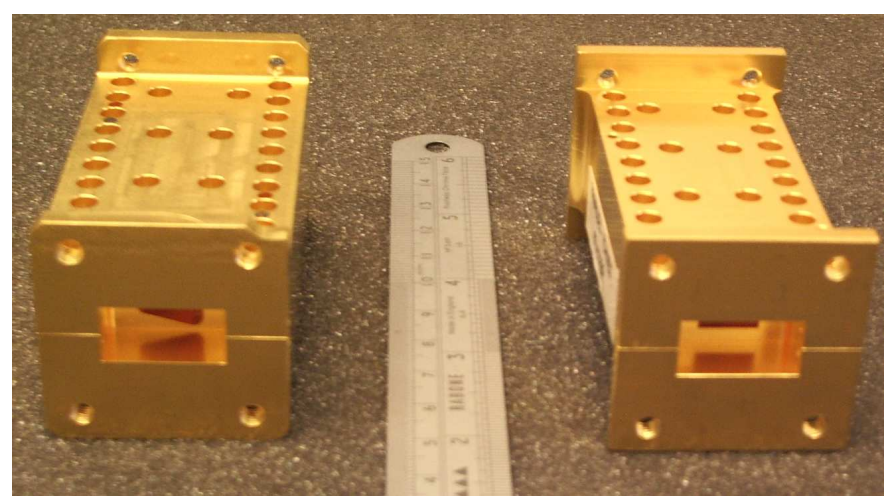

Fig. 11. Photo of the two manufactured gold-plated filters: wedge-shaped filter (left) and quasi-inductive rectangular filter (right).

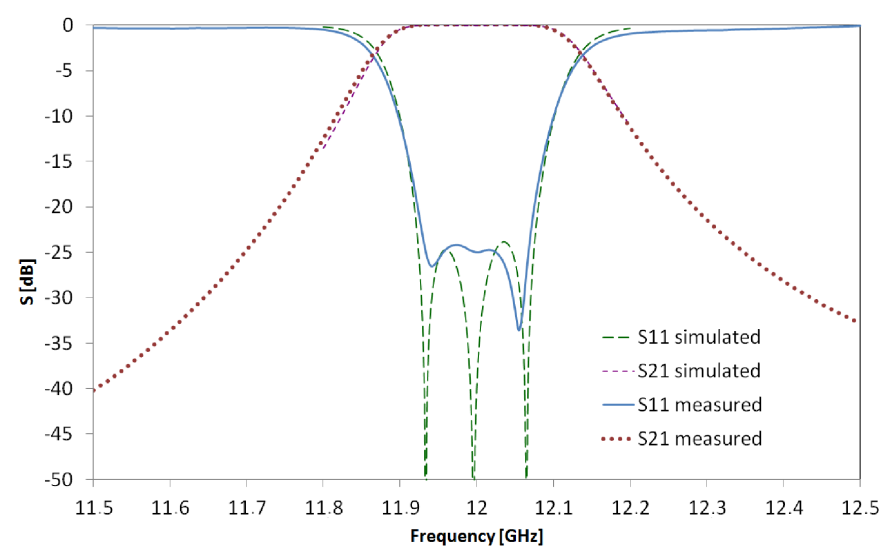

Fig. 12. Comparison between the simulated and measured S-parameters of the gold-plated rectangular filter.

S-parameters is shown in Fig. 12 for the rectangular filter, and in Fig. 13 for the wedge-shaped filter.

The rectangular waveguide filter fulfills almost perfectly the specifications (Table I), just confirming the accuracy of the design process. In the wedge-shaped waveguide case, the measured frequency response is slightly shifted $(\approx 35 \mathrm{MHz})$ towards lower frequencies with respect to the simulated data, probably due to the lower accuracy of the manufacturing process. However, the bandwidth and return losses are according

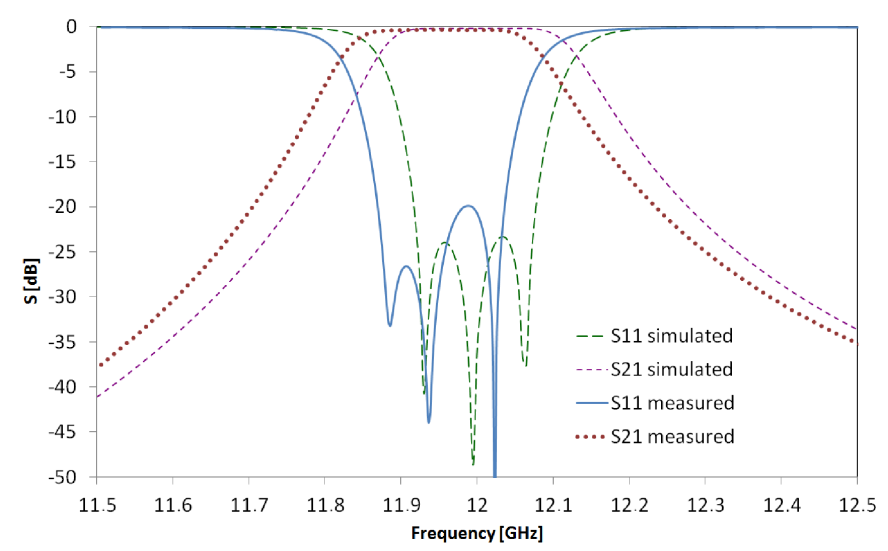

Fig. 13. Comparison between the simulated and measured S-parameters of the gold-plated wedge-shaped filter. 


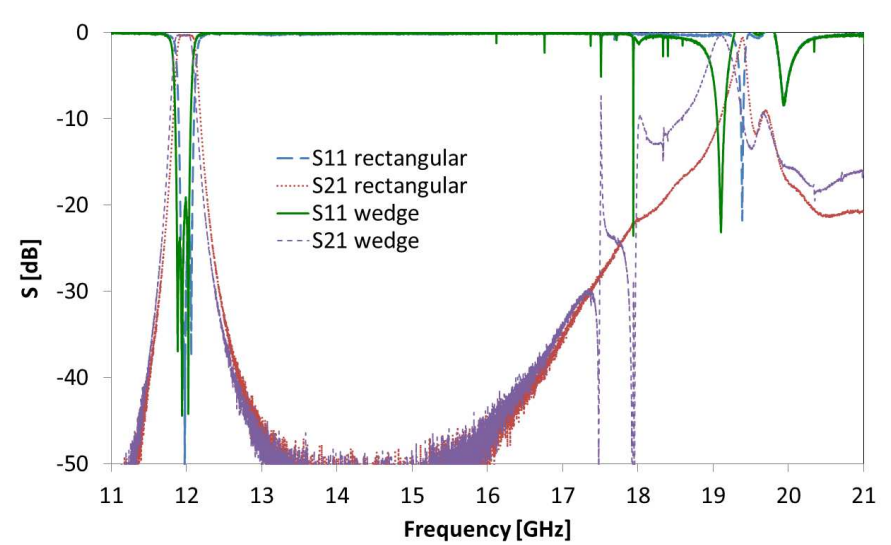

Fig. 14. Measured out-of-band frequency responses of the quasi-inductive and the wedge-shaped gold-plated filters.

to the expected performance, also considering the tolerance analysis described in Section III-A.

The S-parameters have been also measured over a wide frequency range. The objective was to achieve very similar out-of-band performances for both the rectangular and wedgeshaped filters, unlike previous designs (see for instance [6]). The curves are compared in Fig. 14 for the gold-plated pair, and it can be seen that both out-of-band behaviors match quite well, having a repetition of the pass-band at $19.2 \mathrm{GHz}$ and 19.5 GHz (the simulation prediction was around $19.3 \mathrm{GHz}$ ). This result (also confirmed by the aluminum pair) successfully verifies the approach presented in Section II-B. There is a first resonance appearing at $17.4 \mathrm{GHz}$ in the wedge-shaped filter curve, which is related with the higher order modes generated at these frequencies. In a wedge-shaped waveguide with $\alpha=30^{\circ}$, the second and third higher order modes have a lower cutoff frequency than in the rectangular case, which may cause a kind of dual-mode resonance. Another advantage of the approach described in Section II-B is that both filter designs have very similar total lengths (around $74 \mathrm{~mm}$ ).

\section{MP Predictions and Measurements}

All four filters were tested in the Payload Systems Laboratory at ESA-ESTEC. In this way, the accuracy of the prediction tools could be verified under different metallic surface conditions. The gold plating is quite resistant to MP threshold, as we will see in the measurement results, such that no threshold could be detected in some cases for the available power of the test bed. With aluminum, MP discharges could be detected in all cases, which serves better for prediction-tool assessment purposes. Apart from FEST ${ }^{3 \mathrm{D}}$, the ECSS Multipactor Tool [14] was also used for the threshold calculations in the different cases. In this way we can compare the relative prediction accuracy.

The same test-bed was configured for the four experiments, and was similar to the one described in [5], but at a frequency of $12 \mathrm{GHz}$ (in the wedge-shaped case, $11.965 \mathrm{GHz}$ accounting for the slight frequency shift observed in Fig.13). Each measurement started by placing the device under test in the vacuum chamber, close to the radioactive source to
TABLE IV

MP THRESHOLD PREDICTIONS AND MEASUREMENTS AT $12 \mathrm{GHZ}$ (MEASUREMENT LIMIT $6.5 \mathrm{KW}$ )

\begin{tabular}{|l|c|c|c|}
\hline \multirow{2}{*}{ Filter } & \multicolumn{3}{|c|}{ Thresholds } \\
\cline { 2 - 4 } & ECSS MP Tool & FEST $^{3 \mathrm{D}}$ & Measured \\
\hline \hline $\begin{array}{l}\text { Rectangular } \\
\text { (gold) }\end{array}$ & $1300 \mathrm{~W}$ & $1500 \mathrm{~W}$ & $1400 \mathrm{~W}$ \\
\hline $\begin{array}{l}\text { Rectangular } \\
\text { (aluminum) }\end{array}$ & $580 \mathrm{~W}$ & $900 \mathrm{~W}$ & $950 \mathrm{~W}$ \\
\hline $\begin{array}{l}\text { Wedge-shaped } \\
\text { (gold) }\end{array}$ & $1600 \mathrm{~W}$ & no detection & no detection \\
\hline $\begin{array}{l}\text { Wedge-shaped } \\
\text { (aluminum) }\end{array}$ & $700 \mathrm{~W}$ & $3000 \mathrm{~W}$ & $1800 \mathrm{~W}$ \\
\hline
\end{tabular}

ensure sufficient seeding electrons to initiate a MP breakdown. Four MP detection methods were used in each experiment: forward/reverse power nulling and third harmonic detection as global methods, and electrons generation and photons emission as local procedures [15]. Then, the chamber was sealed and pumped for outgassing during 24 hours, until reaching a pressure $<1 \cdot 10^{-7}$ mbar at ambient temperature. The RF source was configured in pulsed mode, with a $2 \%$ duty cycle and a pulse repetition frequency of $1 \mathrm{kHz}$; the maximum achievable RF input power was $6.5 \mathrm{~kW}$. The MP test was started with an input power level of $500 \mathrm{~W}$ and the power was then increased in $20 \mathrm{~W}$ steps. The dwell time at each power level was $10 \mathrm{~min}$. Once MP was detected, the power steps were reduced to $10 \mathrm{~W}$.

All results are summarized in Table IV. The first column corresponds to the predictions with the ECSS MP Tool, and the second one to the results obtained with $\mathrm{FEST}^{3 \mathrm{D}}$ [5]. The form of the secondary emission coefficient (SEEC) function follows the model proposed in [16], where the maximum SEEC value $\left(\delta_{\max }\right)$ is 2 and 2.98 , the first crossover of the electron energy $\left(E_{1}\right)$ is $30 \mathrm{eV}$ and $23.3 \mathrm{eV}$, and the energy at maximum SEEC $\left(E_{\max }\right)$ is $165 \mathrm{eV}$ and $150 \mathrm{eV}$, for gold and aluminum respectively. The SEEC at low energy has the value 0.5 for both materials. The threshold values in $\mathrm{FEST}^{3 \mathrm{D}}$ have been obtained after 50 impacts of each effective electron against the metallic walls. It has to be noted that the predictions with the ECSS MP Tool for the wedge-shaped filter are mere approximations, since a "parallel-plate" model-based prediction [15], [17], [18] is artificially applied to a different geometry. It is assumed that the gap distance is equal to the section length of the critical radius identified in Section II-A, which in this case is around $7.6 \mathrm{~mm}$. The last column of Table IV offers the experimental results.

Excellent agreement is obtained for the rectangular filter threshold values between the $\mathrm{FEST}^{3 \mathrm{D}}$ and the measurement results for both metallic wall surfaces: around $1400 \mathrm{~W}$ with gold and $950 \mathrm{~W}$ with aluminum. This means an improvement with respect to the ECSS MP prediction, especially with the aluminum filter. When considering the gold-plated wedgeshaped filter, interesting consequences can be extracted. Although the ECSS MP prediction suggest a threshold value of $1600 \mathrm{~W}$, no sustained electron resonance could be detected with $\mathrm{FEST}^{3 \mathrm{D}}$ : in no case the electron growth was sufficient to trigger the simulation threshold for MP determination. In 
fact, the measurements verified this last prediction, since no discharge could be detected for the whole available test-bed power range. In the aluminum wedge-shaped filter, discharges were measured at $1800 \mathrm{~W}$ input power. The approximation obtained with the ECSS MP Tool is very conservative. The threshold of $\mathrm{FEST}^{3 \mathrm{D}}$ is $3000 \mathrm{~W}$, closer to the measurement of $1800 \mathrm{~W}$, but still relatively far away from the measurement. The simulations show that the accumulated SEEC values of resonant electrons in wedge-shaped waveguides are more unstable than in the rectangular case. In fact, when reducing the number of impacts of each effective electron, e.g. down to 20 , while keeping the total number of launched electrons, the predicted threshold decreases to $2500 \mathrm{~W}$, whereas in the rectangular filter the threshold value is less sensitive to the maximum number of impacts of each electron. Additionally, the high MP order due to the large gap [19] can explain this discrepancy with the measurements. It should be also noted that, recently, a new MP mode has been discovered [20], which extends the region of parameter space for MP growth, and whose consideration might improve the MP prediction accuracy.

Finally, the excellent MP performance of the wedge-shape filters with respect to the rectangular filters has to be remarked. In the case of aluminum, there is an increase of around $3 \mathrm{~dB}$ in the threshold, beating the results of the first wedge-shaped filter attempts [6], and confirming the success of the design updates. MP has even been completely suppressed within the available experimental capabilities in the gold-plated wedgeshaped filter, as correctly predicted.

\section{CONCLUSIONS}

Having successfully verified the improvements related to the wedge-shaped filter design proposed in this paper, a set of steps can be defined for designing a wedge-shaped waveguide filter with optimal resistance to the MP effect:

1) Determine the dimensions of a rectangular waveguide appropriate for the application, according to the operating frequency and transfer function requirements.

2) Choice of the inclination angle $\alpha$. Optimum is at $30^{\circ}$, but lower $\alpha$-values are possible if other constraints are present. This inclination sets the upper limit for the improvement (as suggested by Fig. 2).

3) Choice of the minimum height $h_{1, \min }$ of the smallest side-wall in order to obtain the desired Q factor without exceeding the manufacturing tolerances.

4) Derive the wedge-shaped resonator-waveguide width, such that the $f_{\mathrm{C}}$ value is equal to the one of the equivalent rectangular waveguide. This value is slightly larger than the reference one. In this way, an appropriate out-of-band frequency response is ensured.

5) Obtain the filter dimensions based on the specifications by means of a synthesis method.

6) Calculate the theoretical trapped-trajectory range, as proposed in Section II-A and in [5].

7) Perform the MP-prediction analysis at radii close to this position in the center of the inner resonator of the device in order to obtain the power and equivalent-voltage MP thresholds. Alternatively, when a susceptibility map such as the one in [5] is available for a given material, an approximated estimation of the power and voltage thresholds can be read from it, after applying suitable voltage-magnification factors.

The MP-threshold measurement of the aluminum wedgeshaped filter has demonstrated an improvement of $3 \mathrm{~dB}$ with respect to the quasi-inductive rectangular one, and the complete suppression of this effect in the gold-plated case. The trade-off is obviously related to a slightly higher design complexity, as it has been described in this paper. Including the results of [6], the advantage of the novel topology has been verified in filters with metallic walls of three different materials: gold, silver and aluminum.

The FEST ${ }^{3 \mathrm{D}}$ MP-prediction tool is not extremely accurate for the wedge-shaped filter cases, as it was justified in the previous section, but is still valuable to have an approximated indication of the MP threshold. Nevertheless, the basis is set for the development of microwave devices that include this kind of wedged waveguide topology in critical gaps, with the goal to avoid the MP breakdown, or to shift it to higher power levels.

\section{ACKNOWLEDGMENT}

The authors would like to thank Ministerio de Ciencia e Innovación (MICINN), Spain, which has supported this work under research project TEC2010-21520-C04-01/TCM. We acknowledge also Mr. Axel Zafra, who adjusted the designed filter dimensions in order to consider the rounded corners of the milling process. The support of the Payload Systems Laboratory (TEC-ETL) of ESA-ESTEC is also appreciated.

\section{REFERENCES}

[1] J. R. M. Vaughan, "Multipactor," IEEE Trans. Electron Devices, vol. 35, no. 7, pp. 1172-1188, July 1988.

[2] V. Semenov, E. Rakova, D. Anderson, M. Lisak, and J. Puech, "Multipactor in rectangular waveguides," Physics of Plasmas, vol. 14, no. 033501 , March 2007.

[3] E. Chojnacki, "Simulation of a multipactor-inhibited waveguide geometry," Physical Review Special Topics - Accelerators and Beams, vol. 3, no. 3, p. 032001, March 2000.

[4] V. Semenov, E. Rakova, N. Zharova, D. Anderson, M. Lisak, and J. Puech, "Simulations of the multipactor effect in hollow waveguides with wedge-shaped cross section," IEEE Transactions on Plasma Science, vol. 36, no. 2, pp. 488-493, April 2008.

[5] J. Hueso, C. Vicente, B. Gimeno, V. E. Boria, S. Marini, and M. Taroncher, "Multipactor effect analysis and design rules for wedgeshaped hollow waveguides," Transactions on Electron Devices (TED), vol. 57, no. 12, pp. 3508-3517, December 2010.

[6] J. Hueso, D. Raboso, D.Schmitt, V. E. Boria, B. Gimeno, and C. Vicente, "Study of the multipactor effect in bandpass wedge-shaped waveguide filters," Transactions on Electron Devices (TED), vol. 58, no. 9, pp. 3205-3212, September 2011.

[7] J. Hueso, D. Raboso, and D. Schmitt, "Microwave waveguide filter with non-parallel plates," Patent, 2005, publication number: 2890787 ; identifier: ESA/PAT/520; filing date: 12-09-2005; publication date: 0506-2009.

[8] P. Overfelt and D. White, "TE and TM modes of some triangular cross-section waveguides using superposition of plane waves," IEEE Transactions on Microwave Theory and Techniques, vol. 34, no. 1, pp. 161-167, January 1986.

[9] Aurorasat S.L., "Full-Wave Electromagnetic Simulation Tool 3D v.6.6," June 2012. [Online]. Available: http://www.fest3d.com 
[10] G. Conciauro, M. Guglielmi, and R. Sorrentino, Advanced Modal Analysis: CAD Techniques for Waveguide Components and Filters. Hoboken, New Jersey: John Wiley and Sons, 1998.

[11] S. Cogollos, S. Marini, V. E. Boria, P. Soto, A. Vidal, H. Esteban, J. V. Morro, and B. Gimeno, "Efficient modal analysis of arbitrarily shaped waveguides composed of linear, circular, and elliptical arcs using the BIRME method," IEEE Trans. Microwave Theory Tech., vol. 51, no. 12, pp. 2378-2390, 2003.

[12] F. Vanin, D. Schmitt, and R. Levy, "Dimensional synthesis for wideband waveguide filters," IEEE MTT-S International Microwave Symposium Digest, vol. 2, pp. 463-466, June 2004.

[13] P. Soto, E. Tarín, V. Boria, C. Vicente, J. Gil, and B. Gimeno, "Accurate synthesis and design of wideband and inhomogeneous inductive waveguide filters," Trans. on Microwave Theory and Tech., vol. 58, no. 8, pp. 2220-2230, August 2010.

[14] ESA-ESTEC and Aurorasat S.L., "ECSS Multipactor Tool." [Online]. Available: http://www.aurorasat.es/multipactortool.php

[15] ECSS Secretariat, "ECSS-E-20-01A: Space engineering - Multipaction design and test," ESA, Tech. Rep., May 2003.

[16] C. Vicente, M. Mattes, D. Wolk, H. Hartnagel, J. Mosig, and D. Raboso, "Multipactor breakdown prediction in rectangular waveguide based components," IEEE MTT-S Int. Microwave Symp. Dig., June 2005.

[17] A. Woode and J. Petit, "Diagnostic investigations into the multipactor effect, susceptibility zone measurements and parameters affecting a discharge," ESA- Working paper No.1556, Tech. Rep., November 1989.

[18] A. Hatch and H. Williams, "The secondary electron resonance mechanism of low-pressure high-frequency gas breakdown," Journal of Applied Physics, vol. 25, no. 4, pp. 417-423, April 1954.

[19] R. A. Kishek, Y. Y. Lau, L. K. Ang, A. Valfells, and R. M. Gilgenbach, "Multipactor discharge on metals and dielectrics: Historical review and recent theories," Physics of Plasmas, vol. 5, pp. 2120-2126, 1998.

[20] R. A. Kishek, "Ping-pong modes: a new form of multipactor," Physical Review Letters, vol. 108, no. 35003, pp. 1218-1221, 2012.

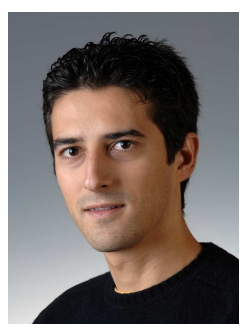

Jaime Hueso Jaime Hueso received the M.S. degree in telecommunication engineering from the Universidad Politécnica de Valencia, Valencia, Spain, in 2003.

From 2004 to 2005, he was a microwave engineer with the European Space Agency, Noordwijk, The Netherlands, where he researched on microwave filter design, high-power testing, and multipactor effect on spaceborne microwave waveguides. From 2006 to 2011, he was with the German Aerospace Center (DLR), Wessling, Germany, involved in the calibration of synthetic aperture radar (SAR) satellite missions like TerraSARX, TanDEM-X, and GMES Sentinel-1. During 2012 he was back in the European Space Agency, supporting future Earth observation technology projects. Since the end of 2012, he has been with the European Patent Office (EPO), Rijswijk, The Netherlands, as patent examiner in the field of antennas.

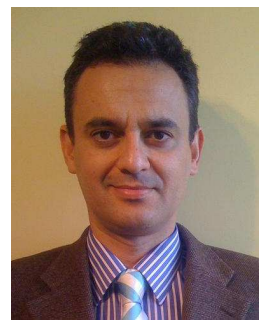

David Raboso David Raboso was born in Alcázar de San Juan, Spain, in 1967. He received the M.S. degree in physics from the University Autónoma of Madrid, Madrid, Spain, in 1992. Since 1992, he has been in the field of RF breakdown and passive intermodulation with the European Space Agency (ESA), Noordwijk, The Netherlands, he is currently the Chairman of European Networks and European Cooperation for Space Standardization (ECSS) Working Groups in multipactor effect, corona, RF high power, and passive inter-modulation. He is also responsible for the research and development and testing services area in RF breakdown with the ESA. Since 2010, he is the Manager of the European highpower RF laboratory based in Valencia, Spain. Mr. Raboso has participated in the organization of every MULtipactorCOronaPIM workshop since 1993 and became the organizer chairman of the event in 2003.

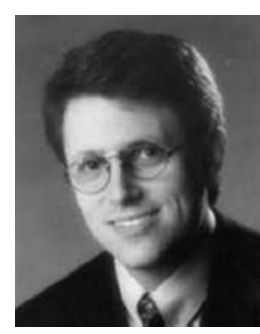

Christoph Ernst Christoph Ernst (M'96) received the Dipl.-Ing. degree in electrical engineering from the University of Dortmund, Dortmund, Germany, in 1996, and the Ph.D. degree from the Institute of Microwaves and Photonics, University of Leeds, Leeds, U.K. in 2001. He is a Member of Staff with the European Space Agency, ESTEC, Holland. His current field of interest is novel techniques, technologies, and the design of microwave passive products for satellite- based systems.

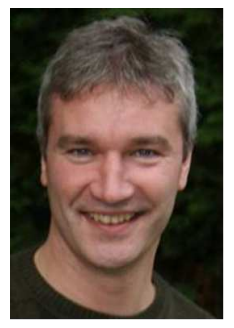

Dietmar Schmitt Dietmar Schmitt received the Ph.D. degree in electrical engineering from the University of Stuttgart, Stuttgart, Germany, in 1995. In 1996, he joined Tesat-Spacecom, Backnang, Germany, as a Manager for the development of passive components such as multiplexers, filters, isolators, and switches for space applications. He joined the European Space Agency (ESA), Noordwijk, The Netherlands, in 2002. He is currently the Head of the Payload Technology and Product Section in the Telecommunication Technologies, Products and Systems Department, European Space Research and Technology Centre of ESA. In this function, he supports European and Canadian payload technologies and products for telecom applications such as antennas, feeds, receivers, passive components (filters, multiplexers, switches, isolators, and harness), digital processors, amplifiers, and payload architectures. He is the author or coauthor of more than 40 publications and the holder of several patents. Dr. Schmitt has been serving as a member of the Technical Program Committee 11 of the IEEE Microwave Theory and Techniques Society, since 1999.

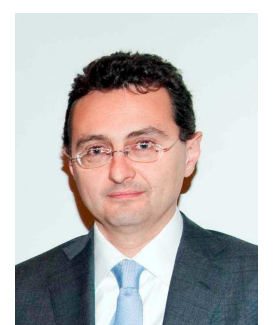

Vicente E. Boria Vicente E. Boria (S'91A'99SM'02) was born in Valencia, Spain, on May 18, 1970. He received the "Ingeniero de Telecomunicación" (with first class honors) and "Doctor Ingeniero de Telecomunicación" degrees from the Universidad Politécnica de Valencia, Valencia, in 1993 and 1997, respectively. In 1993, he joined the Departamento de Comunicaciones, Universidad Politécnica de Valencia, where he has been a Full Professor since 2003. In 1995 and 1996, he was holding a Spanish Trainee position with the European Space Research and Technology Centre of the European Space Agency, Noordwijk, The Netherlands, where he was involved in the area of electromagnetic analysis and design of passive waveguide devices. He is the author or coauthor of 7 chapters in technical textbooks, 75 papers in refereed international technical journals, and more than 150 papers in international conference proceedings. His current research interests are focused on the analysis and automated design of passive components, left-handed and periodic structures, as well as on the simulation and measurement of power effects in passive waveguide systems. Dr. Boria has been a member of the IEEE Microwave Theory and Techniques Society (IEEE MTT-S) and the IEEE Antennas and Propagation Society since 1992. He is a member of the Editorial Boards of the IEEE TRANSACTIONS ON MICROWAVE THEORY AND TECHNIQUES, IEEE MICROWAVE AND WIRELESS COMPONENTS LETTERS, Proceeding of the Institution of Engineering and Technology (Microwaves, Antennas, and Propagation), IET Electronics Letters and Radio Science. He is also a member of the Technical Committees of the IEEE-MTT International Microwave Symposium and of the European Microwave Conference. 


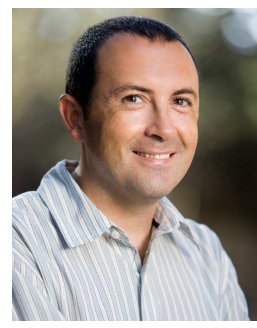

Benito Gimeno Benito Gimeno (M'01) was born in Valencia, Spain, on January 29, 1964. He received the Licenciado degree in physics and the Ph.D. degree from the Universidad de Valencia, Valencia, Spain, in 1987 and 1992, respectively. He was a Fellow at the Universidad de Valencia from 1987 to 1990. Since 1990, he has been serving as an Assistant Professor with the Departamento de Física Aplicada y Electromagnetismo and Instituto de Ciencia de Materiales, Universidad de Valencia, where he became an Associate Professor in 1997 and a Full Professor in 2010. He was with European Space Research and Technology Centre of the European Space Agency, Noordwijk, The Netherlands, as a Research Fellow during 19941995. In 2003, he obtained a Fellowship from the Spanish Government for a short stay at the Universita degli Studi di Pavia, Pavia, Italy, as a Visiting Scientist. His current research interests include the areas of computer-aided techniques for analysis of microwave and millimeter-wave passive components for space applications, waveguides, and cavity structures, including dielectric objects, electromagnetic band-gap structures, frequency selective surfaces, and nonlinear phenomena appearing in power microwave subsystems and particle accelerators (multipactor effect, corona effect, and passive intermodulation phenomena).

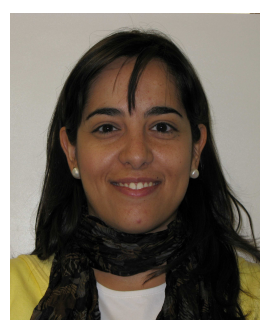

Máriam Taroncher Máriam Taroncher (S'03) was born in Lliria, Valencia, Spain, on October 8, 1979. She received the Telecommunications Engineering degree from the Universidad Politécnica de Valencia (UPV), Valencia, Spain, in 2003. From 2002 to 2004, she was a Fellow Researcher with the UPV in the field of analysis methods for waveguide structures, and is currently working towards the Ph.D. degree at UPV.

Since 2004, she has been a Technical Researcher in charge of the experimental laboratory for high power effects in waveguide devices at the Research Institute iTEAM, UPV, integrated in the Val Space Consortium (VSC). In 2006 she was awarded a Trainee position at the European Space Research and Technology Centre, European Space Agency (ESTEC-ESA), Noordwijk, The Netherlands, where she worked in the Payload Systems Division Laboratory in the area of Multipactor, Corona Discharge and Passive Intermodulation (PIM) effects.

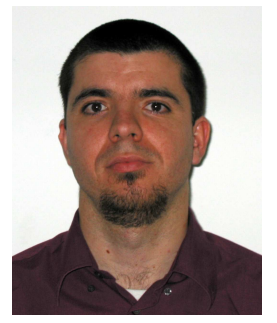

Carlos Vicente Carlos Vicente (M'08) was born in Elche, Spain, in 1976. He received the Dipl. degree in physics from the Universidad de Valencia, Valencia, Spain, in 1999, and the Dr.-Ing. degree in engineering from the Technical University of Darmstadt, Darmstadt, Germany, in 2005. From 1999 to the beginning of 2001, he was a Research Assistant with the Department of Theoretical Physics, Universidad de Valencia. From 2001 to 2005, he was a Professor Assistant with the Institute of Microwave Engineering, Technical University of Darmstadt. Since 2005, he has been with the Microwave Applications Group, Universidad Politécnica de Valencia, Valencia, Spain. In 2006, he cofounded Aurora Software and Testing S. L., which is devoted to the telecommunications sector. His research concerns the analysis and the design of passive components for communication satellites with a special emphasis on high-power practical aspects such as passive intermodulation, corona discharge, and multipaction. 